\title{
Discussion - Normal and active OB stars as extreme condition test beds
}

\author{
summarized by Marc Gagné ${ }^{1}$ and Eduardo Janot Pacheco ${ }^{2}$ \\ ${ }^{1}$ West Chester University, USA \\ ${ }^{2}$ Universidade de Sao Paulo, Brazil
}

\section{Discussion}

Guillaume Dubus: As far as I can tell your model is exactly the same as standard models for high-mass x-ray binaries, the on difference is that you have a mechanism for generating $\gamma$ rays. My question is, we know that there are dozens of other systems that are just LS 5039, so why are they not emitting $\gamma$ rays?

Stan OwOCKI: I don't know. Atsuo, do you know?

Atsuo OKAZAKI: With this accretion rate, you have some accreting power so then a jet is emitted. Of course we cannot know why only these four systems show persistent $\mathrm{TeV}$ emission.

DuBus: But we should know.

Doug GIES: I have a comment and then a question. In Cyg X-1 when the black hole is seen through the wind of the supergiant, you do see these little blips the in the X-ray light curve, so this is a fantastic way of deducing the clumping in the O supergiant.

Owocki: Cyg X-1 did have some high-energy emission, but I don't think it reached $\mathrm{TeV}$ energies, and was only a brief detection with Fermi. This is something that Rich Townsend, David Cohen, and I were going to try to model.

GIES: The question I had was about Bondi-Hoyle accretion. In the picture that you showed of Atsuo's model, you could see matter going in, and the scale was too small a scale to follow. But the cartoon showed that there was an accretion disk. So any idea how much time would you need to build up an accretion disk? To make jets, you would probably need an accretion disk.

OwOCKI: I put the accretion disk there to provide some contrast to the black hole. In the innermost scale, the accretion goes through some innermost scale, but we don't resolve it.

OKAZAKI: The specific angular momentum of the accreting matter is very, very small. The circularization radius is $10^{-5}$ to $10^{-6}$ times the simulation axis, so it's very close to the black, I think.

OwOCKI: That's why the accretion time scale is very small [compared to the simulation time]. I'm not a modeler of accretion at this point, but maybe I'll become one, I don't know. So I can't really answer the question, but it's a simplifying assumption we're 
making at this point just to get a result out. But the time scales are very short because the accretion region is on the size scale of a back hole radius.

JOACHIM PULS: In your last cartoon picture of a jet interacting with a clump, wouldn't the jet destroy the clump?

Owocki: Yes, but in the meantime, it makes $\gamma$ rays. You don't care if it destroys the clump. You just want to see if the interaction with wind of the high-energy particles from the jet will make $\gamma$ rays. That was a big objection the referee had and we had to go back and forth with him a couple of times.

Puls: Maybe I was the referee!

Thomas Rivinius: Joachim mentioned that he has only two stars [HD 64760 and $\gamma$ Ara, that are] rapidly rotating B supergiants. Just from my observational experience, I would like to add another one that is usually classified as a classical Be star. I took a spectrum of it, and decided I'm not interested, and put it in a drawer. I should have known it was interesting. It is $\kappa$ Aquilae, which if you look at its optical spectrum and its IUE spectrum, you see that it is identical to $\gamma$ Ara: it really is a rapidly rotating B0 Ib, with these two little emission bumps left and right. So it would be interesting to look at.

Myron Smith: I want to compliment Maria Fernanda-Nieva's work that she displayed for us this morning on getting fundamental parameters for normal B stars and her spectral synthesis work. I also wanted to call her attention to the publication of a B-star far-UV spectral atlas last winter, in which I look at HST, IUE, FUSE and Copernicus spectra, and the main point here is not the atlas itself, but the line identification work I did for three spectral types. I identified every known line from the atomic line databases. This would be a very good tool for her to use to extend her work into the UV, to see if God knows she can identify any continuum.

MARIA-FERnANDA NiEva: Yes, actually, when I started to update the model atoms for the NLTE spectral synthesis, we already started to do the comparisons in the UV, but this is something we need to continue. But the problem in the UV is that you have so many lines that it is difficult to set the continuum. So you really have to model all the lines. In the visual it's easy because you have individual lines, and you can model the abundances independently, but here we have to model everything together.

M. Smith: Let me add in that case that the intent of the line identification is to only use those lines and no others, because the others make insignificant contributions to the FUV spectra.

GiEs: I had a question for Nathan Smith about $\eta$ Carinae. Nathan in your talk you mentioned this very intriguing possibility that the binary got very close at periastron and dove right into the other star just prior to the great eruption. I was just curious that you said that no merger occurred, but on the other hand it's a fast rotator. Do you think angular momentum might have been transferred?

Nathan Smith: Sure.

GAGnÉ: You really want to go home, don't you? Perhaps we can go back to the first talk 
we heard this morning. Joachim, you told us we should go back and look at $\gamma$ Ara. Can you remind us why this star is important?

Puls: So it seems that my message didn't come completely along. There are two things actually going on in this rotating star business: the first is this polar versus equatorial enhancement of mass loss. Most stars appear not to have this enhancement. The other question is now when we get close to the $\Omega-\Gamma$ limit where the prediction is that the total mass-loss is increasing, this is important for those people doing stellar evolution and this has not been measured or observed so far, and there is only a very small chance to see this really because you need to see a certain signal above a normal mass-loss rate, and only these two stars, and one other perhaps, have the properties, according to scaling laws, to be observable.

Gagné: And what is the luminosity of $\gamma$ Ara?

PULS: Just a regular B supergiant, $\log L$ of 5 or 5.5 .

Sergio Simon-Diaz: I would like to stress again what Fernanda nicely showed us in her talk about the determination of the parameters in the B stars and abundance, because this can affect our interpretation about these stars. For example, I recently analyzed the stars in the Orion OB1 association. These are four subgroups with different ages. In the past, the abundances were obtained and it was found that the youngest stars near the Orion Nebula were oxygen rich, and this was interpreted as a signature of supernova contamination of the new generation of stars, and so on and so forth. Now, taking into account all these details for the abundances, finally I find that the abundances are homogeneous, so there is no trend with oxygen abundance. So, the details of the analysis are important for our interpretation. I want to stress again the importance of doing things properly.

GAGNÉ: In your opinion, why were the previous abundance determinations incorrect?

SimON-DiAz: Because the stellar parameters were derived using photometric indices, and the stellar atmosphere models were not as good as those we have now. These two things together led to the apparent oxygen abundance effect.

NiEva: I just want to comment on the previous work by Cunha \& Lambert that found the abundance anomalies. I did part of my Ph.D. with Katia Cunha, so I know how the work was done in detail. This was my starting point in improving the parameter and abundance determinations of B stars. There are many factors actually. Mainly $\log g$ was derived using $\mathrm{H} \gamma$ profiles in LTE. You get different $\log g$ values in non-LTE. For $T_{\text {eff }}$ you get different values if you use non-ionization equilibria, instead of photometric indices. Many factors contribute: the line selection, the input atomic data of the model atoms. There are many sources of systematic error. It is so difficult to trace back problems in the whole work. They did their best at the time and now there are better techniques, atomic data, computers, so we can do a better job.

GAGNÉ: I think one of the things I took away from your talk though was that there was a rather narrow abundance range that is somewhat consistent with the solar neighbourhood abundance. So, if we are studying the OB stars in open clusters, can we use the the take the effective temperatures and bolometric corrections that you've determined for 
a given spectral type, and bootstrap them onto our photometric samples, provided we have spectral types?

NiEvA: Actually, I've written a paper on this. The conclusion is that photometry needs some kind of calibration. For example you could use the photometric indices from my multi-ionization equilibria effective temperature calibration, but you have different trends for different evolutionary states of the stars. The ZAMS calibration has one curve but if the star is more evolved, you'll have a different curve. But when you have begin analyzing one star, you don't know $\log g$; you don't know its evolutionary state, so you make an initial guess about $T_{\text {eff }}$ and $\log g$. One conclusion of the paper is that you can make a first guess, but you have to take into account the error bar. Whether or not you should interpolate $T_{\text {eff }}$ and $\log g$ depends on the application. For example, will errors at the 20 percent level provide useful constraints? In our work, we needed something like 1 percent accuracy. So, it depends on the application, I think.

PULS: I think that future developments in all these respects will provide more and more spectral libraries available, mostly on the net. We are just now finalizing these new developments and though we might not publish all the line lists we use ourselves, we could make available grids for the most commonly used lines, with respect to a $T_{\text {eff }}$ and $\log g$. So if an observer can at least get decent spectroscopy of a couple of those stars then you can easily derive these parameters.

NiEva: The tools are there. There are many published libraries and the tools are there. The thing is that its very tricky to know how they can be used.

Puls: But not all have the same degree of reliability.

NiEva: Yes, you're right, but tests that can be done. For example, we compared a sample of my work and the work of Sergio. Though we use completely different hydro codes, completely different atomic data, and we sometimes use different lines, we obtain similar results. Perhaps others can use these papers and all the publicly available libraries to perform further tests on the same spectra.

SimON-DiAz: To comment about the calibration and determination of stellar parameters, it's true that maybe the grids can be used as a first approach. But this is dangerous because the calibration depends on the models. If use parameters derived with one model and then you go to another stellar atmosphere model, then you introduce differences up to one thousand [degrees] which can significantly affect the derived abundances. For example, in the tests that we have done, the parameters we derived with ATLAS and in detail with FASTWIND were different. But, in the end, the abundances were the same. So if you do your analysis consistently from start to finish, you can obtain parameters which agree within the error bars. If, however you begin to mix things up, this can be dangerous. Also, nowadays we are fortunate to have very high quality spectra for B stars, for example, because we can investigate the behavior of lines of different ions. For example, I discovered that Si II lines often used for the temperature determination in B2 dwarfs, that is the 41, 28, 30 lines, are not correct in the atomic models. So stellar parameters derived from only these lines are bound to be incorrect. So if you have more lines, you can better constrain all of these things.

N. Smith: I suppose I can expand upon my answer to Doug's question a little bit. When a companion star goes plunging into an extended envelope, inside the envelope of the 
primary star, you know it's gong to be pretty complicated. So tides could probably spin up (add angular momentum) to the star, but another question that clearly there is also a huge amount of mass lost, right? I mean, that could mean you add angle momentum to the envelope, but then that entire envelope is ejected. I mean, we lose ten solar masses, so the net effect of transferring angular momentum is not clear. Another interesting question that goes along with that though is how does this interaction and mass loss, if it's episodic mass loss, how does that affect the orbit? If you lose mass, imagine you have an eccentric orbit, and you're losing mass steadily in a really strong wind, but at a quasi-steady state, you're going to lose most of the mass when you're moving slowly at apastron, that'll act to circularize and widen the orbit, but if you lose mass preferentially at a burst right at periastron, you're going to make the orbit more eccentric each time. And so, $\eta$ Car is currently seen with eccentricity of 0.9 or 0.95 , so it's very hard to get that way without losing mass right at periastron. This is probably important for forming these Wolf Rayet WC colliding wind binaries, the ones that are very eccentric. It's very hard to get that eccentric without doing something like this.

GAGnÉ: Can I ask you to speculate, or anyone to speculate, on the status of the companion star in $\eta$ Car?

N. Smith: A lot of people have been working on this. It's not entirely clear, but the constraints come mainly from the models of the x-ray light curve. From that you get the wind speed, mass-loss rate, and that tells you that it's probably a main-sequence O star or maybe a Wolf Rayet star, but what you also have are upper limits on the ionizing photon luminosity from the amount of Lyman continuum that escapes from the nearby nebulosity. From that it looks like it's not quite such a luminous star: it's less than about 35 to 40 solar masses, but it could be much lower, all the way down to main-sequence stars that are 20 solar masses or so. So there's actually quite a large range of parameter space, but it's consistent with being a fairly normal $\mathrm{O}$ star.

GaGNÉ: Like a main sequence O8 or something.

N. Smith: Like a 30 mass O star. The way, I always describe it is that it's like you take the star that powers the Orion Nebula and you hide it inside the wind of the LBV, so you can't even see it. It's really pretty spectacular.

DieTRICH BAADE: You showed us how the periastron passages line up with certain features in the light curve of $\eta$ Car. Is this perhaps also a chicken-and-egg situation? On the one hand, it could be, as you said, that the outbursts were triggered by a periastron passage. But it could also be that the outburst was already ongoing, but the companion was diving into the first ejector interacting with it somehow and therefore the star was brightening up. Is that also another possibility?

N. Smith: Yeah, the picture I was trying to create, is that if you look at the light curve over several hundred years, you see that, over several hundred years, the star is slowly getting brighter, since the 1600s. That picture suggests that you start out with a primary star a lot like what you have now and it's pretty compact and there's no direct interaction between the two. But as something inside the star is driving itself to start an S Doradus-type outburst, the star is getting cooler and bigger on its own, and through that, as it gets bigger, it's more likely each time to interact with the companion as its radius increases. Maybe that's a feedback loop, maybe not, I don't know. But something is driving the star. This eruption is a long-term process - it's driven into that phase 
where we finally, in the 1820 s and 1830 s, start seeing these peaks, because the radius has gotten to some critical point, where now the companion is really having some effect, it's either digging a hole in its primary, or it's disrupting the primary so that it allows stored thermo energy envelope to escape, I don't know, but something had to happen in the primary to get it there in the first place.

Owocki: Yeah, so, I think about a year and a half ago, when I visited Nathan, we were talking about this just in the context of the current binary, and, because the primary has such a massive wind, I mean, maybe as high as $10^{-3}$ solar masses, the radius of the apparent photosphere, the wind photosphere, can be quite large and, at periastron at least, there's a good chance that, at least, you're carving a cavity, what we call the borehole effect, and my student, Tom Madura just finished his thesis modeling $\eta$ Car's spectrum for HST and light curves is able to reproduce a lot of the optical variations with this cavity model, this borehole model. But obviously the giant eruption, that effect will be even much more dramatic. As far as the angular momentum transfer is concerned, if what we are seeing in $\eta$ Carina, the mass loss is just a larger star because of its wind then it won't be that dynamically coupled onto the actual core hydrostatic star, so i think that one of the opening questions is, in terms of angular momentum transport, is what is the actual size of the hydrostatic core of the star which is thermodynamically coupled? If you distort the wind, you're not going to change the angular momentum of the star, because it's already outflowing at supersonic speeds. So i think it would be very interesting to speculate, or think about, whether during the giant eruption, the hydrostatic core of the star got bigger. That would be probably pretty hard to figure out, because you see the false photosphere so that the optical spectrum, the colors people saw at that time, were form the wind photosphere and not from the core.

GAGNÉ: We will get to you in a second Gloria, but I just want to be clear, I thought in your talk Nathan, though, you were suggesting that when, in the outburst occurred at some point, I'm not sure if it was in the 1840 s, this thing was a hyper-giant. Are you suggesting that it was a hyper-giant at the time of the 1840 event?

N. Smith: So that huge radius I showed was [the one you'd obtain] if you used the present day luminosity and you just made it cooler, so that would be the radius as if it were undergoing an S Doradus outburst, so the radius would have been even bigger. The photosphere would have been even bigger if the luminosity was then increased during the great eruption. What I was trying to show were the conditions as this thing was moving into the great eruption, and so that is the apparent emitting photosphere, the pseudophotosphere in the wind. What that means, whether that's really the photosphere or whether thats a pseudo-photosphere, we're not sure, but, in any case, the outer envelope of the star is very light and fluffy and a very small amount of mass is in that small outer part. So it's not clear.

FERRER: Let me tell you what happened to HD 5980, which I think is a proxy for $\eta$ Carina without all the dust around it that obscures it. HD 5980 is a well-known binary with a 19 day period circular eclipsing binary and between 1980 and 1991 it very gradually became brighter by about 0.6 magnitude and its spectral type became slightly cooler, Wolf Rayet spectral type. There is an Orpheus spectrum of the star 57 days before the first eruption, and that spectrum is no different than the few spectra that we obtained after and, let's say, maybe a year before the eruption. The eruption occurred extremely violently, extremely abruptly. The eruption had a 3 magnitude brightening increase and 
lasted about a year, and then it had spin in it and it quickly came down from the large eruption, but it did not come down all the way. It kept on increasing, so we view this in terms of two different phenomena; you have a long-term brightening, which is analogous of the S Doradus activity phase, which will have gone on, probably, for a long time, had it not been for the presence of the binary companion, so we believe that the binary had triggered the rapid and violent eruption, and thereby changed it's future evolution. It is not declining and we find a 40 year timescale for that. So what we think is happening, is that in HD 5980 the star, for some internal reason, which is what will be very interesting for the stellar evolution people to figure out. for some internal reason, it is increasing its radius very slowly and very gradually, and as Nathan mentioned, and the tidal models we have that show this, as you increase the radius, the interaction effects increase enormously until a critical radius is reached, and the critical radius will depend upon the rotation rate, on the orbital separation, and on the radius of the primary itself, and the mass of the companion, and when that critical radius is reached, it will get the violent eruption. So, I think something similar to this, on different scales, has occurred on $\eta$ Car and I would really urge people to look at HD 5980 as a good proxy for the phenomenon.

Owocki: How much mass was lost in the eruption, Gloria?

FERRER: Approximately $10^{-3}$ solar masses, no more, it lasted, the violent eruption lasted less than a year and, given the velocities and calculations and densities, like $10^{-3}$, but the activity long-term activity stage lasted about 20 years. So that part has had an enhanced mass-loss rate compared to the quiescent state.

OwockI: I think, $10^{-3}$ is maybe more a kin to the kind of mass ejections that, cumulative mass ejections, that you might see in $\mathrm{S}$ Doradus eruptions, right? So maybe, what you're saying is that it removed it before it got a chance to get to the full S Doradus phase, but it wasn't quite the giant eruptions like you have in $\eta$ Carina.

GIES: Just one more comment to follow up Nathan's point about how the ejections in $\eta$ Car are occurring at periastron would increase eccentricity of the orbit. It would seem to me that this would, inevitably, drive the system towards merger at some point, that as you tend towards $e=1$, the stars will crash into each other at some point.

N. Sмiтн: Well, if you take the system we have now, and you have another giant eruption where you lose 10 solar masses at periastron, actually, the system will be unbound and it won't be a binary anymore. 